\title{
Evaluation of in vitro Activities of Tigecycline and Various Antibiotics against Brucella spp.
}

\author{
BETIL OZHAK-BAYSAN ${ }^{1}$, GOZDE ONGUT ${ }^{1}$, DILARA OGUNC ${ }^{1}$, FILIZ GUNSEREN²*, \\ NEVGUN SEPIN-OZEN ${ }^{1}$, FERYAL OZTURK ${ }^{3}$, ORHAN CEM AKTEPE ${ }^{4}$ and MERAL GULTEKIN ${ }^{1}$ \\ ${ }^{1}$ Akdeniz University Faculty of Medicine, Department of Medical Microbiology, Antalya, Turkey \\ ${ }^{2}$ Akdeniz University Faculty of Medicine, Department of Clinical Microbiology \\ and Infectious Diseases, Antalya, Turkey \\ ${ }^{3}$ Anadolu Hospital, Antalya, Turkey \\ ${ }^{4}$ Afyon Kocatepe University Faculty of Medicine, Department of Medical Microbiology, Afyon, Turkey
}

Received 2 July 2009, revised 14 December 2009, accepted 20 December 2009

\begin{abstract}
Brucellosis is a zoonosis with a worldwide distribution and remains a significant public health problem mainly in the developing world. In this study we evaluated the in vitro activities and synergistic effects of antibiotic combinations against blood culture isolates of Brucella spp. In vitro susceptibilities of 76 blood culture isolates of Brucella melitensis and one blood culture isolate of Brucella abortus to doxycycline, streptomycin, gentamicin, trimethoprim-sulfamethoxazole, moxifloxacin, rifampin, ciprofloxacin, and tigecycline were examined by Etest method. For 37 patients with Brucella spp. isolates (36 B. melitensis, 1 B. abortus), antibiotic combinations used for treatment were identified with those tested in vitro for synergy using Etest method. Trimethoprim-sulfamethoxazole and tigecycline were the most active of the compounds tested with $\mathrm{MIC}_{90}$ value of $0.094 \mathrm{mg} / \mathrm{l}$. Among antibiotic combinations only streptomycin-rifampin combination was synergistic for one Brucella spp. isolate. The other antibiotic combinations revealed antagonistic or indifferent activity. Complete clinical response was achieved in all patients. Further studies are required to determine the correlation between the antimicrobial susceptibility and synergy test results with the clinical course of patients. Brucellosis can be adequately treated with existing regimens in our region.
\end{abstract}

K e y w o r d s: brucellosis, Etest for susceptibility, synergy testing, susceptibility, tigecycline

\section{Introduction}

Human brucellosis is a multisystem disease that may involve any organ or system. Successful treatment of brucellosis requires combined antimicrobials with synergy and good intracellular penetration and should be prolonged (Young, 2005). Recently, Centers for Disease Control (CDC) listed Brucella as a potential biological weapon and the European Union (EU) released Bichat guidelines for clinical and bioterrorism related events of brucellosis (Bossi et al., 2004; Yagupsky and Baron, 2005). Moreover, Clinical and Laboratory Standards Institute (CLSI, formerly the National Committee for Clinical Laboratory Standards [NCCLS]) released the first MIC interpretive standards for Brucella spp. in January 2006 (Clinical and Laboratory Standards Institute, 2006). In 1986, the World Health Organization (WHO) recommended a six-week regimen containing doxycycline plus rifampin or doxycycline plus streptomycin for the treatment of brucellosis (World Health Organization, 1986). Most cases respond to standard regimens, with fewer than $10 \%$ manifesting relapses, usually in the first year after treatment. Treatment failures are of concern especially in patients with serious or complicated brucellosis such as spondylitis, neurobrucellosis and endocarditis (Bossi et al., 2004; Pappas et al., 2006b).

Clinical Brucella isolates are generally susceptible to the antibiotic combinations recommended by WHO, however the results of in vitro susceptibility tests do not always predict the clinical outcome. A number of methods used for detection of in vitro synergy between antibiotics have been described (White et al., 1996). The Etest method has been used both for in vitro susceptibility and synergy testing of Brucella spp. as it is less time consuming and less labor intensive. However

\footnotetext{
* Corresponding author: F. Gunseren, Akdeniz University, Faculty of Medicine, Department of Clinical Microbiology and Infectious Diseases, Antalya, Turkey; phone (+90) 2422496762; fax. (+90) 2422272535; e-mail: filizg@akdeniz.edu.tr
} 
synergy testing methods are not yet standardized (Gur et al., 1999; Orhan et al., 2005).

In this study, we evaluated the in vitro activities and synergistic effects of antibiotic combinations against Brucella spp. using Etest method and compared the results with clinical outcome.

\section{Experimental}

\section{Materials and Methods}

A total of 77 Brucella spp. strains isolated at Akdeniz University Hospital and Afyon Kocatepe University Hospital Central Laboratories between September 2001 and June 2006 were tested. The collection included 76 strains of Brucella melitensis and one strain of $B$. abortus. All isolates were obtained from blood using BACTEC 9240 (Becton Dickinson, U.K.) blood culture system. The isolates were collected at the individual study sites and were sent to Akdeniz University Hospital Central Laboratory for identification and susceptibility testing and were stored in stock cultures at $-20^{\circ} \mathrm{C}$ until used. The isolates were identified to the species-level using standard classification tests, including Gram stain, growth characteristics, oxidase activity, urease activity, $\mathrm{H}_{2} \mathrm{~S}$ production, dye sensitivity such as basic fuchsin and thionin and seroagglutination. Identification was confirmed using a semi-nested real-time PCR assay targeting a 223-bp fragment of gene encoding the cell surface protein (BCSP31), specific for the Brucella genus. A class II biological safety cabinet was used to perform the tests.

In the first part of our study, we investigated the minimum inhibitory concentration (MIC) values of antibiotics for all isolates from the two centers by Etest method. In the second part, the patient charts of 37 subjects with Brucella spp. isolates (36 B. melitensis, 1 B. abortus) of the 46 patients from Akdeniz University Hospital, were taken for further assessment. Demographic data, treatment protocol, as well as clinical outcome were evaluated, retrospectively. Each patient's antibiotic combinations used for treatment were identified with those tested in vitro for synergy using E-test method. The results were compared with clinical outcome.

The study was approved by Akdeniz University Medical Faculty Ethical Committee with B.30.2.AKD. 0.01.00.00/Etik/483 protocol number.

Antimicrobial susceptibility tests. The Etest method was performed according to manufacturer's instructions. An inoculum equal to a 1 McFarland turbidity standard was prepared from each Brucella isolate and $10 \mu 1$ of the suspension was inoculated onto Mueller-Hinton agar plates with 5\% sheep blood and Etest strips were applied to the inoculated surface.
The plates were read after $48 \mathrm{~h}$ incubation at $35^{\circ} \mathrm{C}$ under aerobic conditions according to manufacturer's recommendations. MIC values of streptomycin (STR), doxycycline (DOX), rifampin (RIF), gentamicin (GEN), trimethoprim-sulfamethoxazole (SXT), ciprofloxacin (CIP), moxifloxacin (MXF), and tigecycline (TIG) were determined.

Synergy testing. Rifampin-doxycycline, streptomycin-doxycycline, streptomycin-rifampin, trimethoprim-sulfamethoxazole-rifampin, and gentamicindoxycycline combinations were tested against 28 (27 B. melitensis, 1 B. abortus), two, one, five and one Brucella spp. isolates, respectively.

Synergy tests were performed on Mueller-Hinton agar supplemented with $5 \%$ sheep blood by Etest. First, strip A was placed on the inoculated agar surface and left for one hour. The strip's position was marked on the back of the plate. Strip A was removed and strip B was placed on the imprint of A, vertically transposed so $\mathrm{MIC}_{\mathrm{A}}$ and $\mathrm{MIC}_{\mathrm{B}}$ overlap at the same position. Strip $\mathrm{B}$ was left on the agar plate and incubated for $48 \mathrm{~h}$. To evaluate the effect of the combinations, the fractional inhibitory concentration (FIC) was calculated for each antibiotic in each combination. The following formulas were used to calculate the FIC index: FIC of drug A= MIC of drug A in combination/ MIC of drug A alone. FIC of drug $\mathrm{B}=\mathrm{MIC}$ of drug $\mathrm{B}$ in combination/MIC of drug $\mathrm{B}$ alone, and FIC index = FIC of drug A+FIC of drug B. Synergy was defined as an FIC index of $\leq 0.5$. Indifference was defined as an FIC index of $>0.5$ but of $\leq 4$. Antagonism was defined as an FIC index of $>4$.

Quality Control. Quality control isolates, Escherichia coli ATCC 25922 and Streptococcus pneumoniae ATCC 49619 were included in all runs.

\section{Results}

MIC ranges, $\mathrm{MIC}_{50}$ and $\mathrm{MIC}_{90}$ values of the antibiotics for the isolates are shown in Table I. According to their $\mathrm{MIC}_{90}$ values, SXT and TIG were the most active compounds against all Brucella spp. isolates. Among quinolones, MXF demonstrated a lower MIC $\left(\mathrm{MIC}_{50}: 0.125 \mathrm{mg} / \mathrm{l}, \mathrm{MIC}_{90}: 0.25 \mathrm{mg} / \mathrm{l}\right)$ than that of $\mathrm{CIP}\left(\mathrm{MIC}_{50}: 0.19 \mathrm{mg} / \mathrm{l}, \mathrm{MIC}_{90}: 0.38 \mathrm{mg} / \mathrm{l}\right)$. MIC range of RIF was between $0.064-3 \mathrm{mg} / \mathrm{l}$ and a $\mathrm{MIC}_{90}$ value of $1.5 \mathrm{mg} / 1$ for RIF was found. DOX, STR and GEN had good activities against all isolates with a $\mathrm{MIC}_{90}$ value below standard CLSI breakpoints. A complete clinical response was achieved in all patients and no relapses were recorded in the patients' charts.

Synergy test results of antibiotic combinations against Brucella spp. isolates (36 B. melitensis, 1 B. abortus) from 37 patients are shown in Table II. In our study synergy tests were performed using Etest 
Table I

MIC ranges, $\mathrm{MIC}_{50}$ and $\mathrm{MIC}_{90}$ values of antibiotics against Brucella spp. isolates $(\mathrm{n}=77)$

\begin{tabular}{|l|l|l|l|c|}
\hline \multicolumn{1}{|c|}{ Antibiotic } & $\mathrm{MIC}_{50}{ }^{*}$ & $\mathrm{MIC}_{90}{ }^{*}$ & Range & CLSI breakpoint* \\
\hline Doxycycline & 0.047 & 0.125 & $0.016-0.19$ & $\leq 1$ \\
\hline Streptomycin & 0.5 & 1 & $0.064-1.5$ & $\leq 8$ \\
\hline Gentamicin & 0.25 & 0.5 & $0.064-0.75$ & $\leq 4$ \\
\hline Trimethoprim-sulfamethoxazole & 0.047 & 0.094 & $0.016-0.125$ & $\leq 2 / 38$ \\
\hline Moxifloxacin & 0.125 & 0.25 & $0.032-0.25$ & - \\
\hline Rifampin & 0.75 & 1.5 & $0.064-3$ & - \\
\hline Ciprofloxacin & 0.19 & 0.38 & $0.064-0.5$ & - \\
\hline Tigecycline & 0.064 & 0.094 & $0.023-0.5$ & - \\
\hline
\end{tabular}

* MIC values and CLSI breakpoints are expressed in $\mathrm{mg} / \mathrm{l}$.

Table II

Synergy test results of antibiotic combinations against Brucella spp. isolates

\begin{tabular}{|l|c|c|c|c|}
\hline \multirow{2}{*}{$\begin{array}{c}\text { Antibiotic } \\
\text { combinations }\end{array}$} & \multicolumn{4}{|c|}{ Synergy test results n (\%) } \\
\cline { 2 - 5 } & Antagonism & Indifference & Synergy & No of strains \\
\hline RIF-DOX & $25(89.3)^{*}$ & $3(10.7)$ & - & 28 \\
\hline STR-DOX & $2(100)$ & - & - & 2 \\
\hline STR-RIF & - & - & $1(100)$ & 1 \\
\hline SXT-RIF & $3(60)$ & $2(40)$ & - & 5 \\
\hline GEN-DOX & - & $1(100)$ & - & 1 \\
\hline Total n $(\%)$ & $30(81.1)$ & $6(16.2)$ & $1(2.7)$ & $37(100)$ \\
\hline
\end{tabular}

* Twenty-four isolates of B. melitensis and one isolate of B. abortus RIF, rifampin; GEN, gentamicin; SXT, trimethoprim-sulfamethoxazole; STR, streptomycin; DOX, doxycycline.

method. The combination of RIF-DOX yielded antagonism against $89.3 \%$ and indifference against $10.7 \%$ of the isolates. The STR-DOX combination exhibited antagonism against all of the isolates tested. The STRRIF combination was tested in one isolate and was found synergistic. For the combination of SXT-RIF we found antagonism against $60 \%$, and indifference against $40 \%$ of the isolates. The GEN-DOX combination exhibited indifference against one tested isolate (Table II). Demographic data, clinical characteristics of patients and synergy test results of individual patient's antibiotic combinations are presented in Table III.

\section{Discussion}

As shown in several earlier studies, Brucella spp. are generally susceptible to traditional drugs used for the treatment of brucellosis (Bosch et al., 1986; Qadri and Ueno, 1993; Baykam et al., 2004). However strains with decreased susceptibility to RIF and STR have been reported (Baykam et al., 2004; LopezMerino et al., 2004). In our study all strains were susceptible to STR $\left(\mathrm{MIC}_{90}: 1 \mathrm{mg} / \mathrm{l}\right)$ and DOX $\left(\mathrm{MIC}_{90}\right.$ : $0.125 \mathrm{mg} / \mathrm{l}$ ) according to CLSI interpretive breakpoints of $\leq 8 \mathrm{mg} / 1$ and $\leq 1 \mathrm{mg} / \mathrm{l}$, respectively. Furthermore, $\mathrm{MIC}_{90}$ value of STR in our study is lower than the
$\mathrm{MIC}_{90}$ values of previous studies ranging from 2 to $4 \mathrm{mg} / 1$ (Lopez-Merino et al., 2004; Turkmani et al., 2006; Khan et al., 1989; Rubinstein et al., 1991; Akova et al., 1999). However, DOX was found to have a higher $\mathrm{MIC}_{90}$ value when compared to those previously reported (Baykam et al., 2004; Akova et al., 1999; Bodur et al., 2003). Several other studies found even higher $\mathrm{MIC}_{90}$ values for DOX for Brucella isolates (Trujillano-Martin et al., 1999; Yamazhan et al., 2005). GEN had a good activity against all isolates with a $\mathrm{MIC}_{90}$ value below standard CLSI breakpoint. This finding agrees with data from previous studies (Qadri and Ueno, 1993; Turkmani et al., 2006). RIF breakpoints have not been established for Brucella spp. by CLSI. MIC of RIF for our isolates ranges between $0.064-3 \mathrm{mg} / \mathrm{l}$. MIC values of RIF ranging from 0.02 to $16 \mathrm{mg} / \mathrm{l}$ have already been reported (Bosch et al., 1986; Qadri and Ueno, 1993; Lopez-Merino et al., 2004; Turkmani et al., 2006; Khan et al., 1986; Bodur et al., 2003; Al-Orainey et al., 1991). MIC values of SXT ranging from 0.032 to $>32 \mathrm{mg} / \mathrm{l}$ have been reported previously (Bosch et al., 1986; Qadri and Ueno, 1993; Baykam et al., 2004; Lopez-Merino et al., 2004; Turkmani et al., 2006; Khan et al., 1986; Bodur et al., 2003; Al-Orainey et al., 1991). CLSI determined interpretive breakpoints for SXT in 2006 (Clinical and Laboratory Standards Institute, 2006). 
Table III

Characteristics of brucellosis patients and synergy test results of antibiotic combinations

\begin{tabular}{|c|c|c|c|c|c|c|}
\hline $\begin{array}{l}\text { Patient } \\
\text { No }\end{array}$ & $\operatorname{Sex}^{a}$ & $\begin{array}{c}\text { Age } \\
\text { (years) }\end{array}$ & Complications & $\begin{array}{c}\text { Administered } \\
\text { drugs }\end{array}$ & $\begin{array}{c}\text { Synergy } \\
\text { test result }\end{array}$ & $\begin{array}{l}\text { Clinical } \\
\text { outcome }\end{array}$ \\
\hline 1 & $\mathrm{M}$ & 35 & spondylitis & RIF+DOX & $\mathrm{A}$ & Cure \\
\hline 2 & $\mathrm{~F}$ & 59 & spondylitis & RIF+DOX & $\mathrm{A}$ & Cure \\
\hline 3 & $\mathrm{~F}$ & 49 & spondylitis & RIF+DOX & $\mathrm{A}$ & Cure \\
\hline 4 & $\mathrm{~F}$ & 39 & arthritis & RIF+DOX & $\mathrm{A}$ & Cure \\
\hline 5 & $\mathrm{~F}$ & 20 & arthritis & RIF+DOX & $\mathrm{A}$ & Cure \\
\hline 6 & $\mathrm{M}$ & 65 & arthritis & RIF+DOX & $\mathrm{A}$ & Cure \\
\hline 7 & $\mathrm{~F}$ & 44 & arthritis & RIF+DOX & $\mathrm{A}$ & Cure \\
\hline 8 & $F$ & 13 & arthritis & RIF+DOX & $\mathrm{A}$ & Cure \\
\hline 9 & $F$ & 13 & none & RIF+DOX & $\mathrm{A}$ & Cure \\
\hline 10 & $\mathrm{M}$ & 40 & none & RIF+DOX & $\mathrm{A}$ & Cure \\
\hline 11 & M & 46 & none & RIF+DOX & $\mathrm{A}$ & Cure \\
\hline 12 & $\mathrm{~F}$ & 39 & none & RIF+DOX & $\mathrm{A}$ & Cure \\
\hline 13 & $\mathrm{M}$ & 70 & none & RIF+DOX & $\mathrm{A}$ & Cure \\
\hline 14 & $\mathrm{M}$ & 53 & none & RIF+DOX & $\mathrm{A}$ & Cure \\
\hline 15 & $\mathrm{~F}$ & 33 & none & RIF+DOX & $\mathrm{A}$ & Cure \\
\hline 16 & $\mathrm{M}$ & 39 & none & RIF+DOX & $\mathrm{A}$ & Cure \\
\hline 17 & $\mathrm{M}$ & 42 & none & RIF+DOX & $\mathrm{A}$ & Cure \\
\hline 18 & M & 51 & hepatitis & RIF+DOX & $\mathrm{A}$ & Cure \\
\hline 19 & $\mathrm{~F}$ & 26 & abortus & RIF+DOX & $\mathrm{A}$ & Cure \\
\hline 20 & $\mathrm{M}$ & 53 & epididymo-orchitis & $\mathrm{RIF}+\mathrm{DOX}$ & $\mathrm{A}$ & Cure \\
\hline 21 & $\mathrm{M}$ & 24 & none & RIF+DOX & $\mathrm{A}$ & Cure \\
\hline 22 & $\mathrm{~F}$ & 28 & none & RIF+DOX & $\mathrm{A}$ & Cure \\
\hline 23 & $\mathrm{~F}$ & 67 & none & RIF+DOX & $\mathrm{A}$ & Cure \\
\hline 24 & $\mathrm{~F}$ & 27 & neurobrucellosis & RIF+DOX & A & Cure \\
\hline 25 & $\mathrm{~F}$ & 37 & neurobrucellosis & RIF+DOX & A & Cure \\
\hline 26 & $\mathrm{~F}$ & 67 & neurobrucellosis & RIF+DOX & I & Cure \\
\hline 27 & $\mathrm{M}$ & 33 & none & RIF+DOX & $\mathrm{I}$ & Cure \\
\hline 28 & $\mathrm{~F}$ & 27 & arthritis & RIF+DOX & $\mathrm{I}$ & Cure \\
\hline 29 & M & 5 & none & $\mathrm{RIF}+\mathrm{SXT}$ & $\mathrm{A}$ & Cure \\
\hline 30 & $\mathrm{M}$ & 2 & arthritis & $\mathrm{RIF}+\mathrm{SXT}$ & $\mathrm{A}$ & Cure \\
\hline 31 & $\mathrm{M}$ & 2 & arthritis & $\mathrm{RIF}+\mathrm{SXT}$ & $\mathrm{A}$ & Cure \\
\hline 32 & $M$ & 53 & none & $\mathrm{RIF}+\mathrm{SXT}$ & I & Cure \\
\hline 33 & $\mathrm{M}$ & 5 & none & $\mathrm{RIF}+\mathrm{SXT}$ & I & Cure \\
\hline 34 & $M$ & 71 & none & DOX+STR & $\mathrm{A}$ & Cure \\
\hline 35 & $M$ & 66 & spondylitis & DOX+STR & $\mathrm{A}$ & Cure \\
\hline 36 & $\mathrm{~F}$ & 26 & none & RIF+STR & $\mathrm{S}$ & Cure \\
\hline 37 & $F$ & 66 & none & DOX+GEN & I & Cure \\
\hline
\end{tabular}

${ }^{a} \mathrm{~F}$ : female, M: male; ${ }^{b} \mathrm{~A}$ : antagonism, I: indifference, $\mathrm{S}$ : synergism.

RIF: rifampin, GEN: gentamicin, SXT: trimethoprim-sulfamethoxazole, STR: streptomycin, DOX: doxycycline.

All isolates in our study were susceptible to SXT with MIC values ranging from 0.016 to $0.125 \mathrm{mg} / \mathrm{l}$.

An alternative approach may be the use of quinolones for the treatment of brucellosis. Ciprofloxacin and ofloxacin have been used clinically for the treatment of human brucellosis in various combinations. In vitro susceptibility testing results exist for several other quinolones including gatifloxacin, levofloxacin, norfloxacin and pefloxacin (Falagas and Bliziotis, 2006; Pappas et al., 2006a). In our study, we found a $\mathrm{MIC}_{90}$ value of $0.38 \mathrm{mg} / 1$ for CIP which is consistent with data from previous reports (Qadri and Ueno, 1993; Lopez-Merino et al., 2004). Our $\mathrm{MIC}_{90}$ value for MXF $(0.25 \mathrm{mg} / \mathrm{l})$ was lower than those previously reported (0.5-8 mg/l) (Lopez-Merino et al., 2004; TrujillanoMartin et al., 1999; Yamazhan et al., 2005).

Tigecycline is a broad-spectrum glycylcycline antimicrobial agent and seems to have excellent in vitro activity against many Gram-positive and Gram-negative microorganisms (Nathwani, 2005). Pappas et al. 
suggested a shorter duration of treatment with the use of TIG as a single agent (Pappas et al., 2005). $\mathrm{MIC}_{90}$ value of TIG was $0.094 \mathrm{mg} / \mathrm{l}$ for our isolates. There are conflicting data about the MIC of TIG against Brucella in Turkey. In a study of Turan et al. the MIC of TIG was found to be lower than those of RIF and CIP but greater than that of DOX (Turan et al., 2007). TIG was found to be superior to DOX and its $\mathrm{MIC}_{90}$ values were the lowest in the study of Dizbay et al. (2007). This new compound may have a use in the treatment of brucellosis in the future, especially in complicated or serious forms of disease. However it is costly, intravenously administered and its wide use may lead to development of resistance for other serious nosocomial pathogens in countries where brucellosis is endemic (Pappas et al., 2006b).

Time-kill, checkerboard and Etest are most extensively used in vitro methods for detecting synergy (White et al., 1996). There are few reports about in vitro combination studies for Brucellae (Orhan et al., 2005; Rubinstein et al., 1991; Akova et al., 1999). Akova et al. (1999) tested activities of RIF-DOX and STR-DOX by checkerboard method against 20 B. melitensis isolates. They found that RIF-DOX combination was synergistic for 17 isolates, additive for two isolates and indifferent for one isolate. The combination STR-DOX has showed synergistic effect for 18 isolates and was indifferent for two isolates. Dizbay et al. in their study reported TIG-RIF and DOX-RIF combinations demonstrated synergistic activity in all strains. They didn't detect synergy for DOXSTR combination in any strain, but found that $18.75 \%$ of their isolates exhibited antagonism for that combination (Dizbay et al., 2007). On the other hand, Orhan et al. found a high rate of synergy for DOX-STR combination. They also reported that the results of Etest and checkerboard methods agreed with a rate of 55\% in 16 B. melitensis isolates (Orhan et al., 2005). Rubinstein et al. determined in vitro susceptibilities of 86 clinical isolates of $B$. melitensis to minocycline, streptomycin, co-trimoxazole, rifampin and six fluoroquinolones and also studied the possible synergistic effects of several combinations of these antibiotics. They found that no combination exhibited synergy against any of the tested strains by checkerboard test (Rubinstein et al., 1991).

Differences in agreement among synergy testing methods have been reported previously most of which have not been clinically validated. Rubinstein et al demonstrated the discrepancy between the results of checkerboard and time-kill methods in evaluating the antibiotic susceptibility of $B$. melitensis and concluded that time-kill studies have a better correlation with clinical results and therefore may be more appropriate to predict therapy results (Rubinstein et al., 1991). However, Ariza et al. (1986) reported that synergism for the combinations used could not be evaluated appropriately because of very low MICs of tetracycline and DOX for all the isolates. Similarly, all our isolates chosen for synergy testing had very low MICs of DOX, STR, SXT, and GEN according to their CLSI breakpoints. In our study, performance of time-kill studies on the isolate for which synergy was observed as well as some of the antagonistic organism/drug combinations might strengthen our findings with Etest. Further studies are needed to clarify this issue.

In endemic regions, conventional antibrucellar antibiotics are cheap, easy to use and mostly well tolerated. We believe that brucellosis can be adequately treated with existing regimens, with protracted administration of appropriate drug combinations to minimize the percentage of relapses even for the most complicated brucellosis patients.

\section{Acknowledgements}

This study was supported by Akdeniz University Scientific Research Project Unit. Etest strips were provided by Wyeth Pharmaceuticals, Turkey.

\section{Literature}

Akova M., D. Gur, D.M. Livermore, T. Kocagoz and H.E. Akalýn. 1999. In vitro activities of antibiotics alone and in combination against Brucella melitensis at neutral and acidic pHs. Antimicrob. Agents. Chemother. 43: 1298-1300.

Al-Orainey I.O., E.N.S. Saeed, A.M.M. Kambal and M.E. Eltigani. 1991. Sensitivity of Brucella melitensis to chemotherapeutic agents. Saudi. Med. J. 12: 119-120.

Ariza J., J. Bosch, F. Gudiol, J. Liñares, P.F. Viladrich and R. Martín. 1986. Relevance of in vitro antimicrobial susceptibility of Brucella melitensis to relapse rate in human brucellosis. Antimicrob. Agents. Chemother. 30: 958-960.

Baykam N., H. Esener, O. Ergonul, S. Eren, A.K. Celikbas and B. Dokuzoguz. 2004. In vitro antimicrobial susceptibility of Brucella species. Int. J. Antimicrob. Agents. 23: 405-407.

Bodur H., N. Balaban, S. Aksaray, V. Yetener, E. Akýncý, A. Coplan and A. Erbay. 2003. Biotypes and antimicrobial susceptibilities of Brucella isolates. Scand. J. Infect. Dis. 35: 337-338. Bosch J., J. Linares, M.J. Lopez de Goicoechea, J. Ariza, M.C. Cisnal and R. Martin. 1986. In vitro activity of ciprofloxacin, ceftriaxone and five other antimicrobial agents against 95 strains of Brucella melitensis. J. Antimicrob. Chemother. 17: 459-461.

Bossi P., A. Tegnell, A. Baka, F. Van Loock, J. Hendriks, A. Werner, H. Maidhof and G. Gouvras. 2004. Bichat guidelines for the clinical management of brucellosis and bioterrorismrelated brucellosis. Euro. Surveill. 9: 12.

Clinical and Laboratory Standards Institute. 2006. Performance Standards for Antimicrobial Testing, Document M100-S16. Wayne, PA.

Dizbay M., S. Kilic, K. Hizel and D. Arman. 2007. Tigecycline: its potential for treatment of brucellosis. Scand. J. Infect. Dis. 39: 432-434.

Falagas M.E. and I.A. Bliziotis. 2006. Quinolones for treatment of human brucellosis: review of the evidence from microbiologica and clinical studies. Antimicrob. Agents. Chemother. 50: 22-33.

Gur D., S. Kocagoz, M. Akova and S. Unal. 1999. Comparison of Etest to microdilution for determining in vitro activities of 
antibiotics against Brucella melitensis. Antimicrob. Agents. Chemother. 43: 2337-2340.

Khan, M.Y., M. Dizon and F.W. Kiel. 1989. Comparative in vitro activities of ofloxacin, difloxacin, ciprofloxacin, and other selected antimicrobial agents against Brucella melitensis. Antimicrob. Agents. Chemother. 33: 1409-1410.

Lopez-Merino A., A. Contreras-Rodriguez, R. Migranas-Ortiz, R. Orrantia-Gradin, G.M. Hernandez-Oliva, A.T. GutierrezRubio and O. Cardenosa. 2004. Susceptibility of Mexican Brucella isolates to moxifloxacin, ciprofloxacin and other antimicrobials used in the treatment of human brucellosis. Scand. J. Infect. Dis. 36: 636-638.

Nathwani D. 2005. Tigecycline: clinical evidence and formulary positioning. Int. J. Antimicrob. Agents. 25: 185-192.

Orhan G., A. Baykam, Y. Zer and I. Balcý. 2005. Synergy tests by Etest and checkerboard methods of antimicrobial combinations against Brucella melitensis. J. Clin. Microbiol. 43: 140-143.

Pappas G., J. Solera, N. Akritidis and E. Tsianos. 2005. New approaches to the antibiotic treatment of brucellosis. Int. J. Antimicrob. Agents. 26: 101-105.

Pappas G., L. Christou, N. Akritidis and E.V. Tsianos. 2006a. Quinolones for brucellosis: treating old diseases with new drugs. Clin. Microbiol. Infect. 12: 823-825.

Pappas G., P. Papadimitriou, L. Christou and N. Akritidis. 2006b. Future trends in human brucellosis treatment. Expert. Opin. Investig. Drugs. 15: 1-7.

Qadri, S.M. and Y. Ueno. 1993. Susceptibility of Brucella melitensis to the new fluoroquinolone PD 131628: comparison with other drugs. Chemother. 39: 128-131.

Rubinstein E., R. Lang, B. Shasha, B. Hagar, L. Diamanstein, G. Joseph, M. Anderson and K. Harrison. 1991. In vitro sus- ceptibility of Brucella melitensis to antibiotics. Antimicrob. Agents. Chemother. 35: 1925-1927.

Trujillano-Martin I., E. García-Sánchez, I.M. Martinez, M.J. Fresnadillo, J.E. García-Sánchez and J.A. García-Rodríguez. 1999. In vitro activities of six new fluoroquinolones against Brucella melitensis. Antimicrob. Agents. Chemother. 43: 194-195. Turan H., H. Arslan, O.K. Azap, K. Serefhanođlu and H. Uncu. 2007. In vitro antibacterial activity of tigecycline in comparison with doxycycline, ciprofloxacin and rifampicin against Brucella spp. Int. J. Antimicrob. Agents. 30: 186-187.

Turkmani A., A. Ioannidis, A. Christidou, A. Psaroulaki, F. Loukaides and Y. Tselentis. 2006. In vitro susceptibilities of Brucella melitensis isolates to eleven antibiotics. Ann. Clin. Microbiol. Antimicrob. 5: 24.

White R.L., D.S. Burgess, M. Manduru, J.A. Bosso. 1996. Comparison of three different in vitro methods of detecting synergy: time-kill, checkerboard, and Etest. Antimicrob. Agents. Chemother. 40: 1914-1918.

World Health Organization. Sixth Report of the Joint FAO/WHO Expert Committee on Brucellosis. WHO Technical Report Series Rep. 740. Geneva, Switzerland 1986.

Yagupsky, P. and E.J. Baron. 2005. Laboratory exposures to Brucellae and implications for bioterrorism. Emerg. Infect. Dis. 11: $1180-185$.

Yamazhan T., S. Aydemir, A. Tunger, D. Serter and D. Gokengin. 2005. In vitro activities of various antimicrobials against Brucella melitensis strains in the Aegean region in Turkey. Med. Princ. Pract. 14: 413-416.

Young E.J. 2005. Brucella species. pp. 2669-2674. In: Mandell G.L., J.E. Bennett and R. Dolin (eds). Principles and Practice of Infectious Diseases. Churchill Livingstone, Philadelphia. 